\title{
A review of scar assessment scales
}

\author{
Tuyet A Nguyen, ${ }^{1,2}$ Stephanie I Feldstein, MD, ${ }^{1,3}$ Peter R Shumaker, MD ${ }^{4}$ Andrew C Krakowski, MD ${ }^{1,3}$
}

\section{Abstract}

At our current level of understanding, scars are an unavoidable result of disruption of the integument following trauma and other sources of injury in the postnatal period. Millions of people worldwide suffer from diminished quality of life due to varying degrees of disfigurement, functional impairment, and psychosocial comorbidity. Scars also represent a significant financial burden to the healthcare system at large. Substantial momentum currently exists in scar research associated with innovative techniques and devices devoted to treating scars. In order to properly ascertain and compare responses to various therapies, accurate and reproducible qualitative and quantitative assessments are vital. At least 10 different scar assessment scales and tools have been created to date in an attempt to quantify scar severity. However, a "gold standard" scar scale still does not yet exist. A major limitation of most scar scales is their focus on a relatively narrow group of individual subjective and objective features, while failing to address the overall cosmetic, functional, and psychological sequelae. Herein, we provide a brief review of current scar assessment scales, discuss some of the major advantages and limitations of each, and introduce several characteristics that might be addressed in a new "gold standard" scar scale. The assessment and treatment of scars, particularly large traumatic scars, is frequently a multidisciplinary effort. The creation of an "ideal" scar scale will undoubtedly require input from therapists, surgeons, dermatologists, and other professionals alike.

Semin Cutan Med Surg 34:28-36 (c) 2015 Frontline Medical Communications

0 cars do not discriminate. An unavoidable consequence of injury to postnatal human skin from processes such as trauma (eg, burns, lacerations, bites, combat), inflammatory skin disease, and surgical procedures, scars may affect patients of all ages, genders, skin types, socioeconomic levels, and geographic locations. As such, scarring profoundly impacts millions of patients worldwide.

The direct and indirect economic burden associated with scars

${ }^{1}$ Division of Pediatric and Adolescent Dermatology, Rady Children's Hospital, San Diego, California.

${ }^{2}$ Albert Einstein College of Medicine, Bronx, New York.

${ }^{3}$ University of California, San Diego, La Jolla.

${ }^{4}$ Department of Dermatology, Naval Medical Center, San Diego, California.

Disclosures: No funding was secured for this study. The authors have no

financial relationships relevant to this article and no conflict of interest to disclose.

Correspondence: Andrew C. Krakowski, MD; Kids' Scar Treatment and Revision (STAR) Program; Rady Children's Hospital, San Diego; 8010 Frost Street, Suite 602; San Diego, CA 92123. E-mail: akrakowski@rchsd.org and scar management is immense and often underappreciated. There are direct costs including wound care, physical and occupational therapy, and various interventions including pressure garments, topical agents, laser therapy, and surgery. In addition to direct costs, there are harder-to-quantify and potentially much larger costs associated with physical and psychosocial morbidities and loss of productivity. While few studies exist regarding the total costs of scar research and management, it has been estimated that the cost of burn scar management (including inpatient admission, wound care, and follow-up treatment) approaches $\$ 100,000$ per patient. ${ }^{1,2}$ Likewise, current total scar-related expenditures approximates $\$ 12$ billion annually in the United States alone. ${ }^{3}$

Significant momentum currently exists in scar research associated with a proliferation of novel techniques and devices devoted to treating scars and improving the quality of life of scar patients. It is incumbent upon scar experts to make accurate and reproducible clinical assessments so that the evolution of a specific scar can be documented over time in relation to various interventions, and treatment results can be more accurately reproduced, interpreted, and compared on a universal basis. Since the introduction of the Vancouver Scar Scale in 1990, scar rating scales and assessment tools have been developed to help standardize data collection related to scar therapy. More than 10 "scar scales" exist in the literature to date, each attempting to fill a perceived void in assessment. Specific devices may be used to provide quantitative (ie, objective) assessment of the physical attributes of a scar such as changes in height and pliability. Alternatively, patients and providers may provide more qualitative (ie, subjective) assessment that is largely observer-dependent and address features such as color, overall appearance, as well as various comorbidities such as pruritus, pain, and psychosocial sequelae.

Recent advances in minimally invasive techniques such as ablative fractional laser resurfacing (AFR) are leading to consistent and substantial improvements in range of motion and overall function in addition to enhancing the cosmetic appearance and texture associated with scars and scar contractures. ${ }^{4}$ With the emergence of enhanced treatment capabilities, it is increasingly obvious that scar scales devoted primarily to rating individual qualitative visual characteristics such as pigmentation and limited quantitative measurements such as pliability without attention to overall functional impact will be inadequate.

What constitutes the ideal scar scale will provide fertile ground for debate for some time. Any such assessment tool would clearly capture the evolution of a scar through time and through treatment progression. It would also identify all physical and psychosocial comorbidities associated with the scar itself, incorporating objective measurements and overall function. Assessments would be consistently reproducible between users, and documentation would support the utility and goals of any specific services being 
offered as treatment. The scale would not be so brief as to be shortsighted; nor would it be too cumbersome or detail-oriented to preclude its everyday use. Collected data would be fluidly translatable from bedside to bench and back again, helping to directly drive clinical scar research. As a precursor to the development of a more comprehensive scar assessment scale, this manuscript provides a brief review of current scar assessment scales and discusses the relative advantages and potential limitations of each.

\section{Overview of scar scales}

Vancouver Scar Scale and modified Vancouver Scar Scale

Introduced in 1990, the Vancouver Scar Scale (VSS) was the first validated scar scale to be adopted extensively into clinical practice for the assessment of burn scars and remains one of the most frequently used to date..$^{5}$ This scale focuses on four parameters: scar height and thickness, pliability, vascularity, and pigmentation to generate a score ranging from 0 to 13 points. The VSS set a precedent for the systematic evaluation of scars, taking a semiquantitative approach to organize a collection of subjective assessments. However, the VSS has several shortcomings. Multiple studies have demonstrated that the VSS has only indeterminate evidence for validity and reliability, especially with large or irregular scars. ${ }^{6,7}$ Additionally, the VSS does not incorporate symptoms such as pain and itch, and the functional and psychological sequelae of scars.

Due to these limitations, several modifications to the VSS have been proposed in the literature. For example, the original VSS included the pigmentation categories of normal, hypopigmented, and hyperpigmented for 0,1 , and 2 points, respectively. ${ }^{5}$ Based on this system, a hypopigmented scar would have a lower total VSS score than an otherwise identical hyperpigmented scar. Consequently, one introduced modification was the replacement of this scale with an ordinal pigmentation scale, ranging from normal to severely hyper- or hypopigmented. ${ }^{8,9}$ Another important modification by Nedelec et al included the addition of pain and itch parameters. ${ }^{10}$ Forbes-Duchart et al described poor interrater reliability with the VSS when assessing scars in patients with varying skin pigmentation and suggested using a modifier of light, medium, or dark for patients of different skin tones when assessing scars. ${ }^{11}$ These modifications potentially offer some incremental advantages over the original VSS. However, a systematic review by Tyack et al found no resulting improvement in validity, reliability, and responsiveness. ${ }^{7}$ Additionally, subjective parameters beyond itch and pain are not addressed by the VSS and modified VSS.

\section{Seattle Scale}

The Seattle Scale was developed in 1997 by Yeong et al as a photographic scar assessment scale with improved interrater reliability. ${ }^{12}$ The Seattle Scale utilizes a numeric scale in which assessment of several parameters such as surface area, thickness, height, and pigmentation is based on 24 standardized color pictures. Although the Seattle Scale improved interrater reliability, its design allows for negative values for certain parameters such as hypopigmentation or atrophy. While the negative values helped to distinguish different scar types, they also led to "improved" total scores and poor interpretation of scar severity. ${ }^{13}$ This limitation and the lack of attention to symptoms have hindered wide adoption of the Seattle Scale.

\section{Manchester Scar Scale}

Beausang et al introduced the Manchester Scar Scale (MSS) in 1998 with the goal of quantitatively assessing scars based on clinical, photographic, and histological features. ${ }^{14}$ Individual scar attributes including color, contour, radiance, texture, and distortion are evaluated and combined with a visual analog scale to determine an overall score proportional to scar severity. The authors also found a statistically significant correlation between the MSSgenerated assessment and the histologic findings within the scar itself. However, the MSS has been criticized for being better suited to the assessment of linear scars and for a lack of accounting for symptoms. ${ }^{13}$

\section{Hamilton Scale}

The Hamilton Scale is a lesser-known scale that was developed in 1998 specifically as a photographic assessment tool for scars. ${ }^{15}$ Observers are asked to rate several parameters including surface irregularity, thickness, color, and vascularity based on photographs alone. One advantage of this scar scale is its good reliability, even when used by novice observers. Interrater reliability ranged from 0.66-0.90 and test-retest reliability ranged from $0.73-0.89 .{ }^{15}$ Despite this promising reliability, the Hamilton Scale relies on photographs rather than actual scars to drive the assessment process, potentially distorting an observer's interpretation. ${ }^{13}$ Additionally, like the scales preceding it, the Hamilton Scale lacks an assessment of subjective symptoms.

\section{Patient and Observer Scar Assessment Scale}

The introduction of the Patient and Observer Scar Assessment Scale (POSAS) in 2004 was a turning point in the assessment of scars through the use of scales. ${ }^{16}$ The POSAS was the first scale to take into account both the patient and provider perspective, including both a Patient Scar Assessment Scale (P-SAS) and an Observer Scar Assessment Scale (O-SAS). In addition to evaluating the physical characteristics of a scar (eg, vascularization, pigmentation, thickness, relief, and pliability) the POSAS also asks patients to rate any pain and pruritus associated with their scar on a 1-10 ordinal scale. ${ }^{16}$ Draaijers et al compared the reliability and validity of the POSAS to the most frequently used scale, the VSS, and found less variability and greater reliability for single observers. ${ }^{16}$ Though it offers some consideration of subjective features, this is limited to pain and pruritus while ignoring other aspects such as functional deficit and psychological impact. In 2005, the modified POSAS was introduced to provide additional subjective assessment of the impact of the scar on the activities of daily life. ${ }^{17}$

\section{Matching Assessment of Scars and Photographs}

Developed in 2005, the Matching Assessment of Scars and Photographs (MAPS) is a modification of the Seattle Scale designed to aid in the assessment of the long-term progression of a scar and its follow-up. ${ }^{18}$ Similar to the Seattle Scale, the MAPS utilizes a photographic scar assessment scale based on five parameters including border height, thickness, color/pigmentation, surface, and localization. The MAPS improved on the Seattle Scale by introducing set reference photographs and a localization technique to improve interrater reliability during follow-up. ${ }^{18}$ Interrater reliability showed mixed results, with good agreement when assessing border height, thickness, and color (0.55-0.81), but only 
fair when considering surface $(0.25-0.40){ }^{7,18}$ The MAPS offers significant improvements over the Seattle Scale but suffers from some of the same limitations.

\section{Stony Brook Scar Evaluation Scale (SBSES)}

Developed in 2007 by Singer et al, the Stony Brook Scar Evaluation Scale (SBSES) is based on five scar parameters including width, elevation or depression, color, suture or staple marks, and overall appearance. ${ }^{19}$ Each parameter is rated on a scale of 0 to 5, and then added to create a final score. Interrater reliability demonstrated good agreement, ranging from 0.73-0.85. ${ }^{19}$ However, the SBSES lacks a subjective parameter that limits its clinical utility.
University of North Carolina "4P" Scar Scale

The University of North Carolina "4P" Scar Scale (UNC4P) was developed to increase the breadth of qualitative assessment in conjunction with existing scar scales. The "4Ps" of the UNC4P include pain, paresthesias, pruritus, and pliability, and the scale evaluates a scar from a scale of 0 to $12 .^{20,21}$ Although the UNC4P acknowledges the importance of subjective input, it was not designed to be used independently. In the studies conducted by Hultman et al comparing scar characteristics before and after laser resurfacing, the UNC4P was utilized as an adjunct to the traditional VSS. ${ }^{20,21}$ Additionally, the reliability of the UNC4P has yet to be validated.

\section{TABLE Comparison of different parameters included in currently available scar scales}

\begin{tabular}{|c|c|c|c|c|c|c|c|c|c|c|c|c|}
\hline & VSS & $\begin{array}{l}\text { Modified } \\
\text { VSS }\end{array}$ & $\begin{array}{l}\text { Seattle } \\
\text { Scale }\end{array}$ & MSS & $\begin{array}{l}\text { Hamilton } \\
\text { Scale }\end{array}$ & POSAS & $\begin{array}{l}\text { Modified } \\
\text { POSAS }\end{array}$ & MAPS & SBSES & UNC4P & VAS & DLQI \\
\hline \multicolumn{13}{|l|}{ Scar description } \\
\hline \multicolumn{13}{|l|}{ Type } \\
\hline \multicolumn{13}{|l|}{ Age } \\
\hline Surface area & & & - & & & 0 & 0 & & 0 & & & \\
\hline $\begin{array}{l}\text { Height/thickness/ } \\
\text { contour }\end{array}$ & $\bullet$ & $\bullet$ & $\bullet$ & $\bullet$ & $\bullet$ & $\bullet$ & $\bullet$ & $\bullet$ & $\bullet$ & & & \\
\hline Anatomic location & & & & & & & & - & & & & \\
\hline Erythema & ? & - & & & & 0 & 0 & & & & & \\
\hline Pigmentation & 0 & 0 & - & 0 & 0 & 0 & 0 & 0 & 0 & & & \\
\hline \multicolumn{13}{|l|}{$\begin{array}{l}\text { Disruption of anatomic } \\
\text { cosmetic units }\end{array}$} \\
\hline \multicolumn{13}{|l|}{ Scar comorbidities } \\
\hline Psychosocial & & & & & & & & & & & & $\bullet$ \\
\hline Reaction of others & & & & & & & & & & & & - \\
\hline \multicolumn{13}{|l|}{ Hypertrichosis } \\
\hline \multicolumn{13}{|l|}{$\begin{array}{l}\text { Hyperhidrosis/ } \\
\text { hypohidrosis }\end{array}$} \\
\hline Pain & & - & & & & 0 & 0 & & & 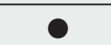 & $\bullet$ & $\bullet$ \\
\hline Pruritus & & - & & & & - & - & & & - & & - \\
\hline Dysesthesia & & & & & & & & & & - & & \\
\hline \multicolumn{13}{|l|}{ Infection } \\
\hline \multicolumn{13}{|l|}{ Lymphedema } \\
\hline \multicolumn{13}{|l|}{$\begin{array}{l}\text { Chronic wound/ } \\
\text { ulceration }\end{array}$} \\
\hline \multicolumn{13}{|l|}{ Skin cancer } \\
\hline $\begin{array}{l}\text { Functional } \\
\text { impairment }\end{array}$ & & & & & & & $\bullet$ & & & - & & - \\
\hline $\begin{array}{l}\text { Overall appearance - } \\
\text { patient }\end{array}$ & & & & & & - & 0 & & & & & \\
\hline $\begin{array}{l}\text { Overall appearance - } \\
\text { observer }\end{array}$ & & & & & & - & ? & & 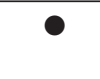 & & & \\
\hline \multicolumn{13}{|l|}{$\begin{array}{l}\text { Amenability to } \\
\text { treatment }\end{array}$} \\
\hline Validity & 0 & 0 & & 0 & & 0 & 0 & & 0 & & 0 & 0 \\
\hline
\end{tabular}


Visual Analog Scale and Dermatology Life Quality Index

Although not specifically designed for scar evaluation, the Visual Analog Scale (VAS) and Dermatology Life Quality Index (DLQI) have become ubiquitous in the evaluation of severity in scars and other dermatologic disorders. The high prevalence of symptoms such as itch and pain and the profound effect of scars on overall quality of life make the use of these subjective scales important to clinical assessment.

The VAS was designed as a simple and quick way to assess patients' subjective experiences of pain. Patients are asked to rate pain intensity by placing a mark on a $100 \mathrm{~mm}$ line ranging from no pain to worst pain imaginable. ${ }^{22}$ The VAS has been validated in several studies, and has been shown to be superior when compared to pain assessment scales such as the fixed interval scale and the verbal rating scale. ${ }^{23-26}$ Although not designed specifically for scar assessment, it remains one of the most frequently used scales for this indication because of its ability to assess a patient's subjective experience with their own scars, which is not typically a parameter included in other scales. ${ }^{27}$

The DLQI was developed in 1994 as the first dermatology-specific assessment of quality of life. ${ }^{28}$ It consists of a questionnaire that evaluates parameters such as pain, itch, embarrassment or social impairment, and functional impairment. It has been shown to have good reliability and validity on several occasions and is useful for assessing quality of life in patients with scars. ${ }^{28-30}$ Like the VAS, the DLQI was not specifically designed to assess scars but is commonly used for this indication because of the impact that a scar may have on a patient's psychosocial health and quality of life.

The frequent use of the VAS and the DLQI in conjunction with other scales for assessment of scars underlies the importance of including subjective input in determining overall scar morbidity. However, no existing scale encompasses a comprehensive review of both objective and subjective features of scars.

\section{Building a more comprehensive scar assessment scale}

The introduction of increasingly patient-centered scales, such as the POSAS and modified POSAS, represents a transition from an emphasis on selected scar features to rating systems poised to assess the overall impact scars have on the patients themselves. However, it is increasingly obvious that the current panel of scar scales and assessment tools are insufficient to guide current multimodality therapy and research for complex scars that includes goals for both cosmetic and functional enhancement. We discuss parameters that are included in the scar scales currently in use (Table) and components we believe might be considered for a more comprehensive scar scale approaching the new gold standard.

\section{Scar type}

No scale to date has included the type of scar in its parameters, primarily due to the fact that most scar scales were developed to assess burn scars. However, it is important to consider that different scar types present with unique natural histories, comorbidities, and treatment challenges. For example, postburn and other traumatic scars may be more likely to present with comorbid conditions such as posttraumatic stress disorder, while dyspigmentation associated with atrophic scars may be more resistant to conventional laser therapy. ${ }^{31,32}$ Also, it has been found that certain scar scales are bet- ter for describing particular types of scars; examples include the VSS, which focuses mostly on burn scars, and the MSS, which is particularly suited for grading linear scars. ${ }^{5,13}$ Therefore, including the type of scar or scar origin may prove useful.

\section{Scar age}

Scar formation and maturation after injury is a dynamic process, with a period of at least 6 to 18 months required for full maturation and somewhat predictable alterations in characteristics such as vascularization and texture. ${ }^{33,34} \mathrm{~A}$ consideration of scar age can inform the evaluation of scar appearance and would be an important topic for research on therapeutic intervention at various time points from injury. For example, ablative fractional carbon dioxide $\left(\mathrm{CO}_{2}\right)$ lasers have been found to be more effective for correcting the abnormal texture and stiffness in mature scars. ${ }^{35}$ Although scar age may be an important factor in treatment response, none of the current scar scales incorporate this parameter.

\section{Area of involvement}

The total surface area of involvement is an important factor to consider when assessing scars. Consider two scars with similar color, pliability, height, and thickness. The first has a surface area of $100 \mathrm{~cm}^{2}$ and the second has a surface area of $10 \mathrm{~cm}^{2}$. Without assessing for surface area, the two scars might be considered equal using multiple scales. However, there may be a significant difference in morbidity solely because of the surface area involved. Furthermore, the area of involvement will also inform issues such as dosing of potential therapies and time required for various procedural interventions. Only three of the scar scales mentioned above take area of involvement into account: the POSAS, the Seattle Score, and the SBSES. ${ }^{12,16,17,19,36}$

\section{Maximum contour irregularity}

Maximum contour irregularity is defined as the maximum distance between the subcutical-dermal border and the epidermal surface of the scar itself. ${ }^{36}$ Height and contour irregularity are important scar parameters because they reflect the amount of hypertrophy or atrophy a scar contains, which can have implications for specific therapies. ${ }^{37}$ All of the previously mentioned scar scales assess scar thickness as a parameter, attesting to its importance, as thicker scars may require more aggressive treatment than thinner scars of the same surface area.

\section{Anatomic location}

The location of a scar is an extremely important parameter in assessing scar severity and associated morbidity, as scars on visible or functional areas may be more concerning to patients. ${ }^{31}$ Patients with visible scars are significantly more likely to face negative reactions from others in public. ${ }^{38}$ In fact, a study of 2,500 members of a national burn survivor support group demonstrated a statistically significant correlation between visible scarring and lower body selfesteem, including perceived stigmatization. ${ }^{39}$ Other studies demonstrated that visible facial disfigurement from burn scars is a risk factor for subsequent depression and posttraumatic stress disorder. ${ }^{31,38}$ Scar formation in certain anatomic locations can cause functional impairment as well, leading to increased morbidity for patients. However, this factor is neglected in most of the current scales. Only the MAPS includes a localization technique in the assessment of scars. ${ }^{18}$ 


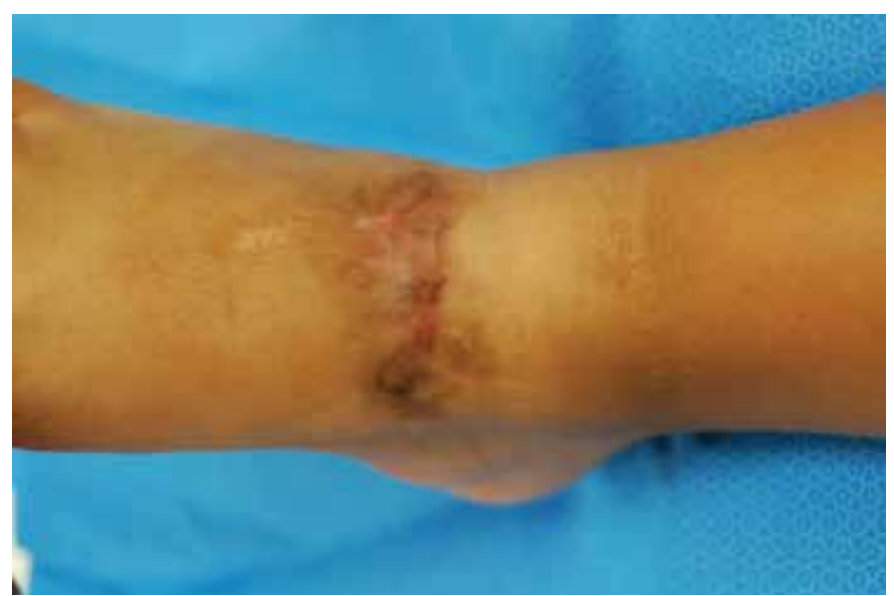

FIGURE 1. This traumatic scar in a pediatric patient with Fitzpatrick Skin Type 4 demonstrates trichromic "mottling" (a combination of hyperpigmentation, hypopigmentation, and persistent erythema), a phenomenon that existing scar scales do not adequately consider.

\section{Erythema}

Erythema is commonly seen in scars, especially immature scars where hyperplasia and/or hypervascularity play a role in the maturation phase of wound healing. ${ }^{13}$ Erythema typically diminishes after several months, but it may persist for years in some cases. Therefore, it is an important parameter to include in a scar scale because it could reflect issues such as the current state of scar maturation, incipient pathological scarring, and potential response to vascular-specific laser devices. The VSS and the POSAS are the only two scales that include vascularity as a specific parameter. ${ }^{5,16}$ However, most scales include color or pigmentation, which can often reflect the presence of dyspigmentation or erythema.

\section{Dyspigmentation}

Pigmentary changes are commonly seen in scars, resulting from disparities in melanocyte density and melanin deposition in scarred versus unscarred skin. ${ }^{13}$ Scarred skin can be hypopigmented, hyperpigmented, or both (ie, mottled) at the same time (Figure 1). This can often be very distressing to patients as the pigmentary contrast of the scar against normal skin may make it much more noticeable. Hypopigmented scars provide an additional complication because relative lack of melanin may leave the skin without a normal level of protection against ultraviolet radiation. ${ }^{40}$ All of the current scar scales, except for the unvalidated UNC4P, include pigmentation as a parameter, indicating its importance in assessing scar severity. However, scales for assessing dyspigmentation face a unique challenge because they must assess for both hypo- and hyperpigmentation. Much of the criticism on pigmentation as a parameter, such as those included in the VSS or Seattle Scale, is that hypopigmentation is often given a negative or lower score which leads to "improved" total scores compared to their hyperpigmented counterparts. ${ }^{5,12}$ Therefore, the need for a more consistent consideration of pigmentation persists.

\section{Anatomic cosmetic units}

Conceptually, certain key anatomic locations can be subdivided into multiple topographical cosmetic units. The face is the location that is most commonly demarcated into cosmetic units that include the forehead, eyes, nose, lips, chin, ears, and neck. ${ }^{34}$ Anatomic cosmetic units are important in scar assessment because a scar that falls in one unit or at the junction lines separating cosmetic units is typically less conspicuous than one crossing the boundary between two or more units. Conversely, scars involving multiple cosmetic units are often more noticeable and may be more likely to cause cosmetic or functional compromise. Therefore, it may be important to consider anatomic cosmetic units in the evaluation of scars, as more noticeable scars can be associated with increased morbidity for patients..$^{38}$ No scar scale to date incorporates anatomic cosmetic units or scar conspicuity as a parameter.

\section{Psychosocial impact}

The majority of the research regarding the psychosocial consequences of scars comes from the burn literature. Burn victims in particular face significant aesthetic disfigurement, which can frequently be more disabling than the physical sequelae because of associated emotional, social, and economic difficulties. ${ }^{41}$ In fact, depression and posttraumatic stress disorder are significant problems for patients with burn scars, with prevalence rates ranging from $13 \%-23 \%$ and $13 \%-45 \%$, respectively. ${ }^{38}$ In addition, a patient's perspective of a scar may be more important in psychosocial outcome variables than the actual physical characteristics of the scar such as total body surface area. ${ }^{39}$ In a study by Fauerbach et al, it was found that patients with worse body image dissatisfaction after burn injury had significantly lower psychosocial and physical adjustment after two years of follow-up, even after controlling for injury severity. ${ }^{42}$

Other psychosocial problems such as sexual dysfunction have been reported in individuals with extensive scars, especially women. ${ }^{38}$ This is thought to be due to alteration in appearance that affects an individual's body image and self-esteem. However, these psychosocial factors are often underreported and underdiagnosed, with few existing studies on these issues. ${ }^{38}$ Although the psychosocial effects of scars can have a huge influence on quality of life, none of the current scar scales address this factor when assessing for scar severity.

\section{Reaction of others to scars}

Patients often experience stigmatization and discrimination in reaction to scars, such as staring, startled responses, intrusive questions, avoidance, rude comments, teasing, and bullying..$^{39}$ It has been shown that even children express negative attitudes toward scarred and disfigured people. ${ }^{41}$ Because of this, scarred individuals can be left with a sense of rejection, loneliness, and social isolation. ${ }^{41}$ For example, patients with burns scars have reported increased difficulty meeting new people, making friends, and developing intimate relationships. ${ }^{38}$ Stigmatization related to scarring has been hypothesized to be a causative factor in the development of three common psychological sequelae after a severe burn: low body image, social anxiety, and depression. ${ }^{38}$ Like the psychosocial results, the stigmatization caused by scarring is often ignored when assessing for scar severity.

\section{Hypertrichosis/hypotrichosis}

Although not commonly reported, alterations in hair density after trauma can be a distressing consequence of scar formation 


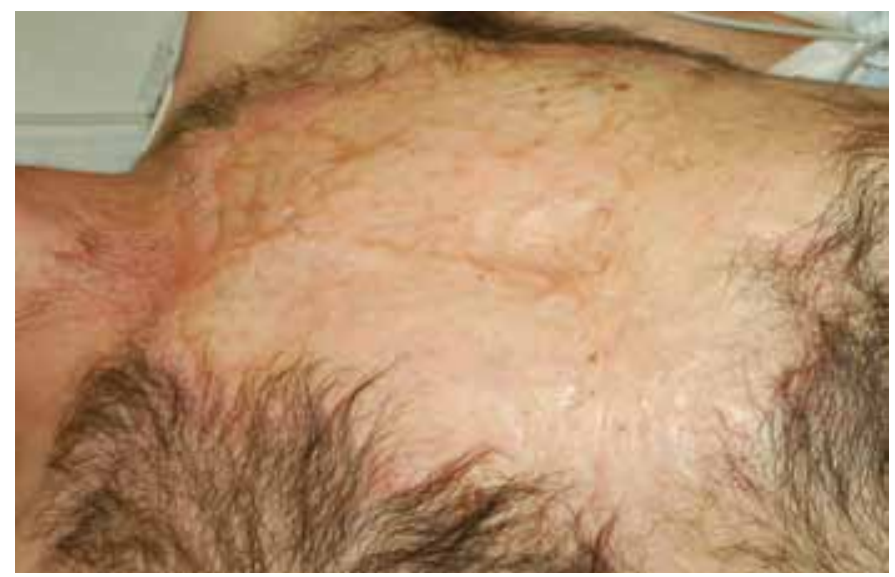

FIGURE 2. This adolescent patient sustained a scald injury to his chest as an infant leaving him unable to perspire or grow hair in the affected areas. Current scar scales do not consider hyperand hypohidrosis or hyper- and hypotrichosis in their assessments.

(Figure 2). Posttraumatic scarring frequently results in alopecia in important cosmetic areas such as the scalp and brow, and hair bundling or entrapment may lead to issues such as pain, folliculitis, and abscess formation. Gupta et al described 18 patients with postsurgical hypertrichosis on their knee replacement surgery scars. ${ }^{43}$ Additionally, hypertrichosis is also found more frequently in patients with orthopedic casts and splints that are utilized in individuals with surgical scars. ${ }^{44}$ It is speculated that the increased vascularity and increased growth factors present in hypertrophic scars can promote the growth of hair. ${ }^{43}$ Currently, no scar scale to date includes hyper- or hypotrichosis as a parameter.

\section{Hyperhidrosis/hypohidrosis}

Hyperhidrosis or hypohidrosis of the skin are relatively common findings after trauma (Figure 2). Injury to the skin and the subsequent process of scarring leads to abnormal deposition of collagen matrix and inferior functional qualities of the skin, with loss of functional cutaneous adnexae such as hair follicles and sweat glands. ${ }^{45}$ Hyperhidrosis is particularly common and problematic in the residual limbs of amputees, adversely affecting the fit and comfort of prosthetic devices. ${ }^{46,47}$ However, these factors are not included in any of the currently available scar scales.

\section{Pain}

Pain is a well-recognized and often-distressing result of trauma and subsequent scar formation. For example, pain (sometimes accompanied by paresthesias) along the sternal scar and in the upper extremities persists in up to $30 \%$ of cases of patients with poststernotomy scars. ${ }^{48}$ Burn scars are also often accompanied by significant pain. ${ }^{38}$ Because of its significant effect on quality of life, the presence of pain plays an important role in deciding whether or not to pursue treatment. Of the current scar scales available, only the modified VSS, POSAS, and the unvalidated UNC4P address this critical issue.

\section{Pruritus}

Pruritus, or itch, is commonly found in individuals with scars. This phenomenon is thought to occur through a variety of fac- tors, including friction, inflammation, stimulation of nerve endings around the scar, and increased levels of $\beta$-endorphin in scar sites. ${ }^{49}$ Interestingly, predictors of pruritus associated with a scar include a high percentage of total body surface area affected, female gender, having undergone a surgical procedure, and early posttraumatic stress disorder. ${ }^{13}$ Additionally, keloid scars are much more likely to have associated pruritus, affecting approximately $86 \%$ of keloid patients. ${ }^{50}$ A retrospective study on 23 patients with burn scars revealed that $87 \%$ of subjects experienced daily pruritus. ${ }^{51}$ Additionally, pruritus was reported as unbearable in $94 \%$ of subjects with chronic itch and $86 \%$ of subjects with acute itch. ${ }^{51}$ Although pruritus contributes to the significant morbidity associated with scarring, it is not a common parameter included in scar scales. Only three scales to date address this important factor: the modified VSS, POSAS, and the UNC4P.

\section{Dysesthesia}

Dysesthesia describes the sensation of unpleasant or abnormal sense of touch, such as anesthesia, burning, wetness, electric shock, and pins and needles. It is thought that these sensations arise from small nerve fiber neuropathy in scarred tissue due to nerve entrapment and physical pressure from collagen deposition. ${ }^{50}$ Dysesthesias are typically unwelcome by patients, and thus should be considered when evaluating for severity. These sensations are common in scar patients, but with the exception of the UNC4P, are not included in current scales.

\section{Infection}

It is accepted that infections such as folliculitis, cellulitis, abscesses, and fasciitis are common complications following injury and subsequent wound healing. However, it has been found that scars and related treatment may be risk factors for infection, as well. A retrospective study by Mukhdomi et al evaluated 55 patients with burn scar cellulitis, showing that therapeutic procedures may actually facilitate infections within scars. ${ }^{52}$ Current infections and a history of infection are important to consider in a scar assessment scale because this may alter prognosis and guide the choice of treatment modalities. However, none of the currently available scar scales include this as a parameter.

\section{Lymphedema}

Localized tissue swelling is a common complication in scars. This process is believed to be multifactorial, occurring through vascularization, fibrosis, and lymphedema. ${ }^{53}$ After trauma, lymphatic disruption and subsequent fibrosis can result in lymphedema. Warren et al demonstrated decreased lymphatic drainage from scar sites through use of a radiotracer. ${ }^{53}$ Lymphedema (ie, impaired lymphatic circulation) is an important parameter to consider in the assessment of a scar because it may influence scar appearance and selection of treatment modalities. However, this parameter is not currently included in any of the available scar scales.

\section{Chronic wounds}

Chronic wounds associated with scarring can present significant challenges to patients and providers alike (Figure 3). It is unclear why certain individuals suffer from chronic wounds and others do not, though factors such as increased tension, skin fragility, and bacterial biofilms likely play a role. Chronic wounds can be 

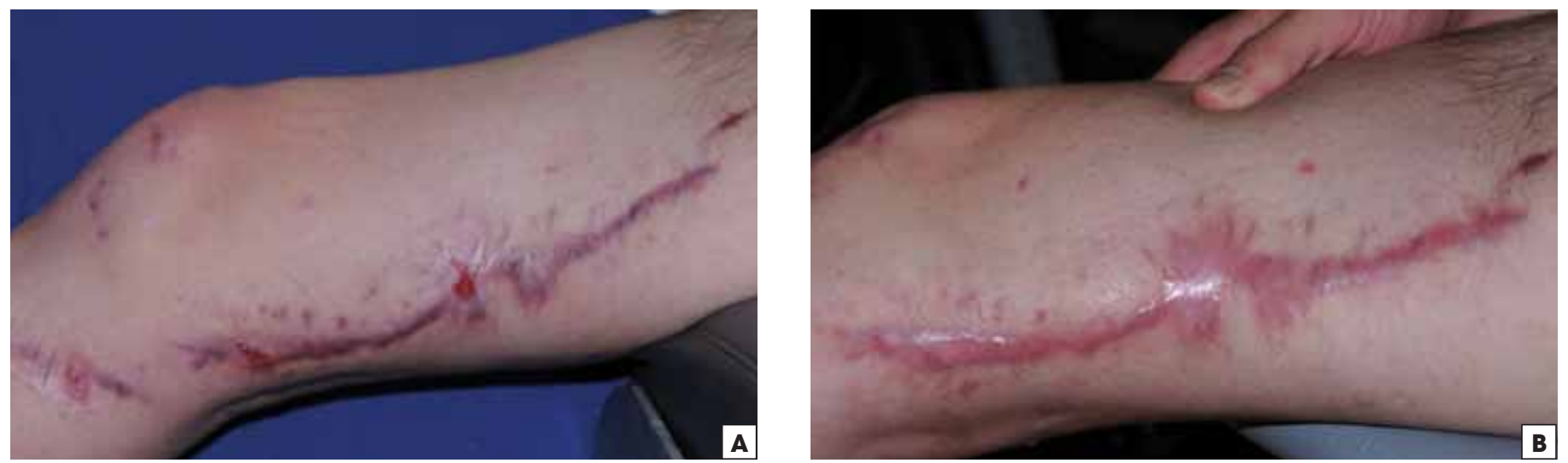

FIGURE 3. A) Chronic wounds are frequently noted in the setting of traumatic scars due to skin fragility, tension, and other factors. B) Rapid healing of two chronic erosions approximately one week after the initial ablative fractional laser treatment.

distressing for affected patients and absorb significant resources in professional wound care and dressings. Furthermore, chronic wounds increase the risk of secondary infections and even malignancy (ie, Marjolin's ulcer).$^{54}$ Despite these issues, none of the current scar scales address this parameter.

\section{Skin cancer}

Skin cancer is one of the most dangerous complications associated with scar formation. It is well known that skin cancers (especially squamous cell carcinoma) occur more frequently in scarred skin, especially burn scars. ${ }^{55-61}$ There have also been reports of increased incidence of basal cell carcinoma and malignant melanoma in patients with scars. ${ }^{56,57}$ Because of the long-term implications of skin cancer for a trauma patient, it is important to assess for presence or absence of skin cancer when treating a patient with scars. However, none of the scar scales to date include skin cancer in their parameters.

\section{Functional impairment}

Probably the most glaring omission from current scar scales is the degree of functional impairment resulting from scar contractures

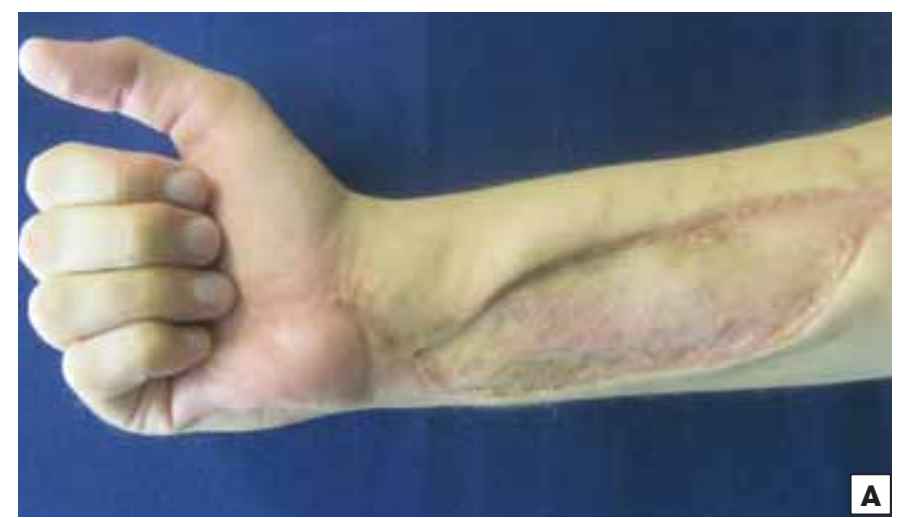

or symptomatic scars (Figure 4). Diminished abilities to ambulate and perform routine tasks of daily living, let alone return to normal work, can have obvious and profound effects on the patient's overall quality of life. ${ }^{23}$ The recent emergence of minimally invasive procedures associated with consistent functional enhancements such as ablative fractional laser resurfacing highlight the need for comprehensive scar scales that account for functional improvement to inform future research and therapeutic comparison., ${ }^{4,58-60}$ The UNC4P references range of motion deficit; however, except for the modified POSAS, no existing validated scar scale assesses the degree of functional impairment caused by a scar.

\section{Conclusion}

Scars are a common problem, often with substantial associated morbidity and economic burden. Multiple scar assessment scales have been developed to help assist in the consistent evaluation of scar severity, progression, and response to treatment. However, no gold standard scar scale exists to date. One of the major limitations of most current scar scales is a focus on a limited number of physical features of individual scars without significant attention to the overall cosmetic, functional, or psychological sequelae. A number

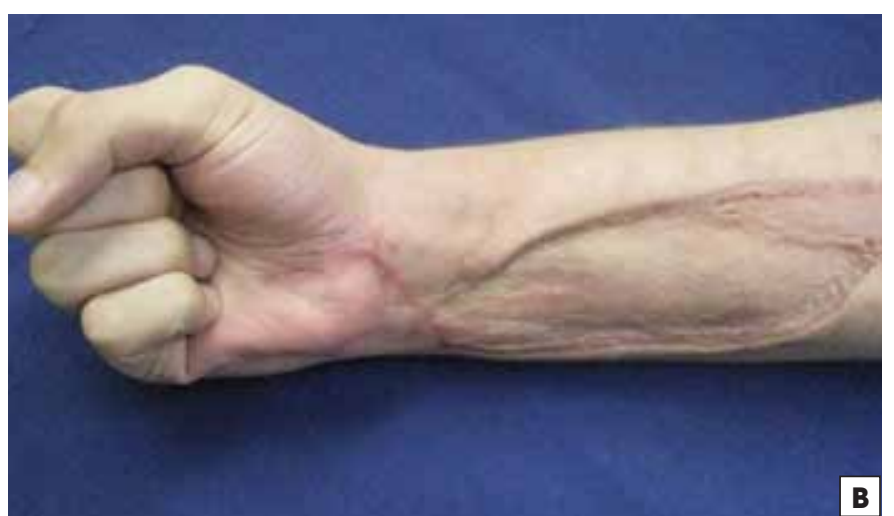

FIGURE 4. Relatively small limitations in range of motion can have a significant impact on overall function, especially involving the upper extremity and hand. A) The patient is attempting to fully flex his fingers approximately three months after a gunshot wound requiring fasciotomy and a split-thickness skin graft for reconstruction. B) Demonstrated herein is the rapid improvement in range of motion approximately two weeks after the patient's initial ablative fractional laser treatment. In the very short interval between the before and after photos in these cases, it is unlikely that there would have been significant differences according to most current scar rating scales. However, there are substantial implications for the patient in terms of function and overall quality of life. 
of scales including the POSAS, modified POSAS, and modified VSS have attempted to incorporate subjective data (pain and pruritus) into scar assessment. While important, this information is insufficient to capture the full impact of scars on a patient's quality of life or the longitudinal effects of emerging multimodal therapies. This manuscript has briefly described a variety of existing scales and their perceived limitations and introduced additional features that should be considered, in the view of the authors, for incorporation into a more comprehensive future scale. It is relatively easy to point out flaws. The hard work remains in creating a scale that is easy to use, integrates both quantitative and qualitative data from a heterogeneous population of patients and scars, and informs the user regarding the aggregate physical and psychosocial impact. Undoubtedly, this task will require a multidisciplinary effort that includes therapists, surgeons, dermatologists, and other professionals engaged in the treatment of these patients.

Disclaimer: The views expressed in this article are those of the authors and do not reflect the official policy or position of the Department of the Navy, Department of Defense, or the U.S. government. Dr. Shumaker is a military service member. This work was prepared as part of his official duties. Title 17, USC, § 105 provides that Copyright protection under this title is not available for any work of the United States Government.Title 17, USC, § 101 defines a U.S. Government work as a work prepared by a military service member or employee of the U.S. Government as part of that persons official duties.

\section{References:}

1. Sánchez JL, Perepérez SB, Bastida JL, Martinez MM. Cost-utility analysis applied to the treatment of burn patients in a specialized center. Arch Surg. 2007;142(1):5057; discussion 57.

2. Pellatt RA, Williams A, Wright H, Young AE. The cost of a major paediatric burn. Burns. 2010;36(8):1208-1214.

3. Sen CK, Gordillo GM, Roy S, et al. Human skin wounds: a major and snowballing threat to public health and the economy. Wound Repair Regen. 2009;17(6):763-771.

4. Anderson RR, Donelan MB, Hivnor C, et al. Laser treatment of traumatic scars with an emphasis on ablative fractional laser resurfacing: consensus report. JAMA Dermatol. 2014;150(2):187-193.

5. Sullivan T, Smith J, Kermode J, McIver E, Courtemanche DJ. Rating the burn scar. J Burn Care Rehabil. 1990;11(3):256-260.

6. Roques C, Teot L. A critical analysis of measurements used to assess and manage scars. Int J Low Extrem Wounds. 2007;6(4):249-253.

7. Tyack Z, Simons M, Spinks A, Wasiak J. A systematic review of the quality of burn scar rating scales for clinical and research use. Burns. 2012;38(1):6-18.

8. Nedelec B, Correa JA, Rachelska G, Armour A, LaSalle L. Quantitative measurement of hypertrophic scar: intrarater reliability, sensitivity, and specificity. J Burn Care Res. 2008;29(3):489-500.

9. Nedelec B, Correa JA, Rachelska G, Armour A, LaSalle L. Quantitative measurement of hypertrophic scar: interrater reliability and concurrent validity. J Burn Care Res. 2008;29(3):501-11.

10. Nedelec B, Shankowsky HA, Tredget EE. Rating the resolving hypertrophic scar: comparison of the Vancouver Scar Scale and scar volume. J Burn Care Rehabil. 2000;21(3):205-212.

11. Forbes-Duchart L, Marshall S, Strock A, Cooper JE. Determination of inter-rater reliability in pediatric burn scar assessment using a modified version of the Vancouver Scar Scale. J Burn Care Res. 2007;28(3):460-467.

12. Yeong EK, Mann R, Engrav LH, et al. Improved burn scar assessment with use of a new scar-rating scale. J Burn Care Rehabil. 1997;18(4):353-355; discussion 352.

13. van der Wal MB, Verhaegen PD, Middelkoop E, van Zuijlen PP. A clinimetric overview of scar assessment scales. J Burn Care Res. 2012;33(2):e79-e87.

14. Beausang E, Floyd H, Dunn KW, Orton CI, Ferguson MW. A new quantitative scale for clinical scar assessment. Plast Reconstr Surg. 1998;102(6):1954-1961.

15. Crowe JM, Simpson K, Johnson W, Allen J. Reliability of photographic analysis in determining change in scar appearance. J Burn Care Rehabil. 1998;19(2):183-186.

16. Draaijers LJ, Tempelman FR, Botman YA, et al. The patient and observer scar as- sessment scale: a reliable and feasible tool for scar evaluation. Plast Reconstr Surg. 2004;113(7):1960-1965; discussion 1966-1967.

17. Fearmonti RM, Bond JE, Erdmann D, Levin LS, Pizzo SV, Levinson H. The modified Patient and Observer Scar Assessment Scale: a novel approach to defining pathologic and nonpathologic scarring. Plast Reconstr Surg. 2011;127(1):242-247.

18. Masters M, McMahon M, Svens B. Reliability testing of a new scar assessment tool, Matching Assessment of Scars and Photographs (MAPS). J Burn Care Rehabil. 2005;26(3):273-284.

19. Singer AJ, Arora B, Dagum A, Valentine S, Hollander JE. Development and validation of a novel scar evaluation scale. Plast Reconstr Surg. 2007;120(7):1892-1897.

20. Hultman CS, Friedstat JS, Edkins RE, Cairns BA, Meyer AA. Laser resurfacing and remodeling of hypertrophic burn scars: the results of a large, prospective, beforeafter cohort study, with long-term follow-up. Ann Surg. 2014;260(3):519-529; discussion 529-532.

21. Hultman CS, Edkins RE, Lee CN, Calvert CT, Cairns BA. Shine on: Review of Laser- and Light-Based Therapies for the Treatment of Burn Scars. Dermatol Res Pract. 2012;2012:243651.

22. Scott J, Huskisson EC. Graphic representation of pain. Pain. 1976;2(2):175-184

23. Joyce CR, Zutshi DW, Hrubes V, Mason RM. Comparison of fixed interval and visual analogue scales for rating chronic pain. Eur J Clin Pharmacol. 1975;8(6):415420 .

24. Ohnhaus EE, Adler R. Methodological problems in the measurement of pain: a comparison between the verbal rating scale and the visual analogue scale. Pain 1975;1(4):379-384.

25. Carlsson AM. Assessment of chronic pain. I. Aspects of the reliability and validity of the visual analogue scale. Pain. 1983;16(1):87-101.

26. Jensen MP, McFarland CA. Increasing the reliability and validity of pain intensity measurement in chronic pain patients. Pain. 1993;55(2):195-203.

27. Bae SH, Bae YC. Analysis of frequency of use of different scar assessment scales based on the scar condition and treatment method. Arch Plast Surg. 2014;41(2):111115 .

28. Finlay AY, Khan GK. Dermatology Life Quality Index (DLQI)--a simple practical measure for routine clinical use. Clin Exp Dermatol. 1994;19(3):210-216.

29. Mazharinia N, Aghaei S, Shayan Z. Dermatology Life Quality Index (DLQI) scores in burn victims after revival. J Burn Care Res. 2007;28(2):312-317.

30. Balci DD, Inandi T, Dogramaci CA, Celik E. DLQI scores in patients with keloids and hypertrophic scars: a prospective case control study [in English, German]. $J$ Dtsch Dermatol Ges. 2009;7(8):688-692.

31. Yu BH, Dimsdale JE. Posttraumatic stress disorder in patients with burn injuries. $J$ Burn Care Rehabil. 1999;20(5):426-433; discussion 422-425.

32. Fife D. Practical evaluation and management of atrophic acne scars: tips for the general dermatologist. J Clin Aesthet Dermatol. 2011;4(8):50-57.

33. Atiyeh BS. Nonsurgical management of hypertrophic scars: evidence-based therapies, standard practices, and emerging methods. Aesthetic Plast Surg. 2007;31(5):468-492; discussion 93-94.

34. Fattahi TT. An overview of facial aesthetic units. J Oral Maxillofac Surg. 2003;61(10):1207-1211.

35. Hultman CS, Edkins RE, Wu C, Calvert CT, Cairns BA. Prospective, before-after cohort study to assess the efficacy of laser therapy on hypertrophic burn scars. Ann Plast Surg. 2013;70(5):521-526.

36. Vercelli S, Ferriero G, Sartorio F, Stissi V, Franchignoni F. How to assess postsurgical scars: a review of outcome measures. Disabil Rehabil. 2009;31(25):2055-2063.

37. Arno AI, Gauglitz GG, Barret JP, Jeschke MG. Up-to-date approach to manage keloids and hypertrophic scars: a useful guide. Burns. 2014;40(7):1255-1266.

38. Van Loey NE, Van Son MJ. Psychopathology and psychological problems in patients with burn scars: epidemiology and management. Am J Clin Dermatol. $2003 ; 4(4): 245-272$

39. Lawrence JW, Mason ST, Schomer K, Klein MB. Epidemiology and impact of scarring after burn injury: a systematic review of the literature. J Burn Care Res. 2012;33(1):136-146.

40. Chadwick S, Heath R, Shah M. Abnormal pigmentation within cutaneous scars: A complication of wound healing. Indian J Plast Surg. 2012;45(2):403-411.

41. Gilboa D, Bisk L, Montag I , Tsur H. Personality traits and psychosocial adjustment of patients with burns. J Burn Care Rehabil. 1999;20(4):340-346; discussion 338-339.

42. Fauerbach JA, Heinberg LJ, Lawrence JW, et al. Effect of early body image dissatisfaction on subsequent psychological and physical adjustment after disfiguring injury. Psychosom Med. 2000;62(4):576-582.

43. Gupta S, Gupta S, Kanwar AJ , Kumar B. Hypertrichosis surrounding scar of knee replacement surgery. J Am Acad Dermatol. 2004;50(5):802-803.

44. Akoglu G, Emre S, Metin A, Bozkurt M. High frequency of hypertrichosis after cast application. Dermatology. 2012;225(1):70-74.

45. Blaha J. Physiology and pathology of skin after burns and derangement of gene expression. Acta Chir Plast. 2006;48(4):127-132.

46. Meulenbelt HE, Geertzen JH, Dijkstra PU, Jonkman MF. Skin problems in low- 
er limb amputees: an overview by case reports. J Eur Acad Dermatol Venereol. 2007;21(2):147-155.

47. Meulenbelt HE, Geertzen JH, Jonkman MF, Dijkstra PU. Skin problems of the stump in lower limb amputees: 1. A clinical study. Acta Derm Venereol. 2011;91(2):173-177.

48. van Leersum NJ, van Leersum RL, Verwey HF , Klautz RJ. Pain symptoms accompanying chronic poststernotomy pain: a pilot study. Pain Med. 2010;11(11):1628-1634

49. Zhu J, Cheng B, Liu H, Tang J, Xiang X, Peng Y. Expression of beta-endorphin in hypertrophic scar and its relationship with pruritus [in Chinese]. Zhongguo Xiu Fu Chong Jian Wai Ke Za Zhi. 2012;26(6):731-734.

50. Lee SS, Yosipovitch G, Chan YH, Goh CL. Pruritus, pain, and small nerve fiber function in keloids: a controlled study. J Am Acad Dermatol. 2004;51(6):1002-1006.

51. Parnell LK, Nedelec B, Rachelska G, LaSalle L. Assessment of pruritus characteristics and impact on burn survivors. J Burn Care Res. 2012;33(3):407-418.

52. Mukhdomi GJ, McCauley RL, Desai MH, Mitchell AT, Herndon DN. Cellulitis associated with burn scars: a retrospective review. J Burn Care Rehabil. 1996;17(4):346-350.

53. Warren AG, Slavin SA. Scar lymphedema: fact or fiction? Ann Plast Surg.
2007;59(1):41-45.

54. Al-Zacko SM. Malignancy in chronic burn scar: a 20 year experience in Mosul-Iraq Burns. 2013;39(7):1488-1491.

55. Bartle EJ, Sun JH, Wang XW, Schneider BK. Cancers arising from burn scars. A literature review and report of twenty-one cases. J Burn Care Rehabil. 1990;11(1):46-49.

56. Kowal-Vern A, Criswell BK. Burn scar neoplasms: a literature review and statistical analysis. Burns. 2005;31(4):403-413.

57. Kikuchi H, Nishida T, Kurokawa M, Setoyama M , Kisanuki A. Three cases of malignant melanoma arising on burn scars. J Dermatol. 2003;30(8):617-624.

58. Perry A, Elston J, Reynolds H, et al. Ablative fractional photothermolysis in the treatment of scar contractures of the wrists and forearms: a retrospective data analysis. J Am Acad Dermatol. 2014;71(4):841-842.

59. Shumaker PR, Kwan JM, Landers JT , Uebelhoer NS. Functional improvements in traumatic scars and scar contractures using an ablative fractional laser protocol. $J$ Trauma Acute Care Surg. 2012;73(2 Suppl 1):S116-S121.

60. Krakowski AC, Goldenberg A, Eichenfield LF, Murray JP, Shumaker PR. Ablative fractional laser resurfacing helps treat restrictive pediatric scar contractures. Pediatrics. 2014;134(6):e1700-e1705. 